\title{
AVALIAÇÃO DA PREVALÊNCIA DA INFECÇÃO POR HELICOBACTER PYLORI EM PACIENTES PORTADORES DE CÂNCER GÁSTRICO
}

\author{
EVALUATION OF THE PREVALENCE OF INFECTION BY HELICOBACTER \\ PYLORI IN PATIENTES WITH GASTRIC CANCER
}

\author{
Marcelo Fernandes Rangel, TCBC-PB ${ }^{1}$ \\ Waldir Pedrosa de Amorim² \\ Maria de Fátima Duques de Amorim ${ }^{3}$ \\ Pedro Duques de Amorim ${ }^{4}$ \\ Leonardo Pires de Sá Nóbrega ${ }^{5}$
}

\begin{abstract}
RESUMO: Objetivo: Nos últimos anos, evidências de associação entre Helicobacter pylori e câncer gástrico têm sido relatadas por inúmeros estudos. Este trabalho objetiva investigar a prevalência da infecção por este microorganismo em pacientes com câncer gástrico, oriundos do Hospital de Câncer Napoleão Laureano (João Pessoa - PB) e determinar o risco relativo para o desenvolvimento desta neoplasia nos pacientes infectados. Método: Com esta finalidade, 16 pacientes com diagnóstico confirmado de adenocarcinoma gástrico foram submetidos à endoscopia digestiva alta para coleta de fragmentos da mucosa gástrica, para realização do teste da urease, sendo então, pareados com um grupo de 16 controles com exames endoscópicos normais. Resultados: Dos 16 portadores de câncer, $28,1 \%$ estavam infectados, versus $25 \%$ para os do grupo controle. Nos indivíduos infectados, houve maior prevalência da infecção nos portadores de lesões gástricas distais (43,8\%), nos tumores Borrmann III (37,6\%), e moderadamente diferenciados $(37,6 \%)$. Houve associação estatisticamente significante entre o grau de diferenciação e a presença da infecção pelo $H$. pylori $(\mathrm{p}<0,10)$. O risco relativo estimado para a associação entre câncer gástrico e infecção pelo $H$. pylori foi de 1,28\% (odds ratio $=1,28 \%$ ). Conclusão: Estes resultados permitem concluir que a infecção pelo $H$. pylori é um fator de risco relativo para o desenvolvimento do adenocarcinoma gástrico.
\end{abstract}

Descritores: Helicobacter pylori; Câncer Gástrico; Infecção; Prevalência

1 Professor Adjunto - Doutor do Departamento de Cirurgia do C.C.S. da UFPB. TCBCD. Ex-Chefe do Serviço de Cirurgia Abdominal do Hospital de Câncer Napoleão Laureano.

2 Ex-Professor de Gastroenterologia do C.C.S. da UFPB; Gastroenterologista do Hospital Universitário da UFPB; Chefe do Endocenter - Unidade de Fígado e Gastroenterologia da Paraíba.

3 Professora Auxiliar do Curso de Nutrição do C.C.S. da UFPB. Médica do Endocenter - Unidade de Fígado e Gastroenterologia da Paraíba.

4 Médico Residente em Gastroenterologia do Hospital das Clínicas da UNESP, Botucatu - SP. Ex-bolsista PIBIC/CNPq/UFPB

5 Médico Residente em Cirurgia Geral do Hospital Universitário Lauro Wanderley da UFPB, João Pessoa - PB. Ex-bolsista PIBIC/ $\mathrm{CNPq} / \mathrm{UFPB}$.

Recebido em 15/05/2002

Aceito para publicação em 18/11/2002

Trabalho realizado nos Serviços de Cirurgia Abdominal do Hospital de Câncer Napoleão Laureano e de Endoscopia Digestiva do Endocenter - Unidade de Fígado e Gastroenterologia - João Pessoa - PB.

Apoio: PIBIC/CNPq/UFPB 


\section{INTRODUÇÃO}

O câncer gástrico é a segunda causa de morte por neoplasia maligna do mundo ${ }^{1}$, sendo superado apenas pelo câncer de pulmão, com aproximadamente 900.000 casos novos diagnosticados a cada ano $^{2}$. É uma afecção de distribuição mundial e de etiologia multifatorial, estando alguns fatores exógenos e endógenos relacionados com esta neoplasia. Idade, sexo, herança familiar, grupo sangüíneo, acloridria, anemia perniciosa, pólipos adenomatosos, úlcera gástrica, fatores sócioeconômicos, etnia, dieta, estômago operado e doença de Ménétrier, estariam correlacionados de diversas formas à esta afecção ${ }^{3}$. Mais recentemente, evidências de associação entre Helicobacter pylori e câncer gástrico têm sido relatadas em inúmeros estudos ${ }^{1,4,5,6}$.

Em 1994, a International Agency for Research on Cancer, World Health Organization (IARC), baseando-se no resultado de nove estudos retrospectivos de casos-controle e quatro estudos cohort, demonstrou uma associação causal entre a infecção por $H$. pylori e o câncer gástrico ${ }^{7}$.

A gastrite atrófica decorrente de vários anos de infecção pelo $H$. pylori funcionaria como lesão precursora da metaplasia intestinal, para o desenvolvimento da neoplasia gástrica maligna ${ }^{8,9,10}$. A probabilidade de desenvolvimento do câncer seria determinada por fatores ambientais como dieta, duração e idade em que foi contraída a infecção, virulência das cepas dos microorganismos e fatores inerentes ao hospedeiro ${ }^{11}$.

Estudos de sorologia para esta bactéria em pacientes portadores de câncer gástrico indicam que um maior risco de desenvolvimento da neoplasia estaria relacionado à infecção pelas cepas CagA do H. pylori ${ }^{12-17}$. Nos últimos anos, o número de casos de câncer gástrico vem diminuindo, provavelmente pelas modificações dietéticas, refrigeração dos alimentos e declínio na infecção pelo $H$. pylori $i^{2}$. Ainda que existam controvérsias a respeito do real valor da erradicação rotineira deste microorganismo, alguns autores admitem que o caminho na prevenção do câncer gástrico esteja no controle da infecção ${ }^{18}$. Entretanto, várias publicações na literatura mostram resultados conflitantes no que concerne à associação da infecção pelo $H$. pylori, idade dos pacientes, localização do tumor, tipo histológico e estadiamento da neoplasia ${ }^{19}$.
O presente estudo tem por finalidade investigar a correlação de infecção por $H$. pylori em pacientes com diagnóstico prévio de câncer gástrico, e comparar com um grupo controle, constituído por indivíduos com endoscopia digestiva alta normal. Objetiva, igualmente, correlacionar a infecção pelo H. pylori com a idade, sexo, localização tumoral, aspecto macroscópico da lesão, e grau de diferenciação histológica.

\section{MÉTODO}

No período de agosto de 1999 a agosto de 2000 foram investigados 16 pacientes portadores de câncer gástrico, provenientes do Hospital de Câncer Napoleão Laureano (João Pessoa-PB) que foram pareados com 16 pacientes controle originários da demanda natural do Serviço de Endoscopia Digestiva do Endocenter - Unidade de Fígado e Gastroenterologia (João Pessoa-PB).

Todos os pacientes selecionados responderam a um questionário que abordava itens de interesse para a avaliação endoscópica. O questionário era complementado durante a realização do exame, momento em que os autores estavam em contato direto tanto com os aspectos referentes à técnica bem como com o aspecto endoscópico da lesão gástrica.

Durante a realização do exame endoscópico, eram colhidos quatro fragmentos gástricos da região tumoral, sendo imediatamente colocados na solução que contém uréia para realização do teste da urease ${ }^{3}$, ao mesmo tempo que outros fragmentos da neoplasia eram enviados para estudo histopatológico.

Em seguida, era realizada a coleta dos fragmentos da mucosa gástrica para realização do teste da urease dos pacientes controle, grupo composto por indivíduos que apresentavam exame endoscópico do trato digestivo superior sem anormalidades.

Quanto ao aspecto macroscópico do câncer gástrico, a classificação selecionada foi a proposta por Borrmann (apud Mincis $)^{19}$, que dividiu os tumores gástricos em: B I (superficial), B II (polipóide, vegetante ou protruso), B III (úlcero-infiltrativo), B IV (infiltrativo e difuso ou linitis plastica), e B V (não classificável segundo os critérios anteriores). Esta classificação fornece elementos úteis para a análise do prognóstico, além de ser simples e de fácil avaliação durante a endoscopia. 
Os dados obtidos foram analisados estatisticamente, e correlacionados com as variáveis presentes no questionário: sexo, idade, localização do tumor, classificação de Borrmann, grau de diferenciação histológica e teste da urease. As técnicas estatísticas utilizadas foram o teste do Qui-Quadrado, regressão logística, odds ratio (razão de chance), medida de associação $\mathrm{V}$ de Cramer e intervalo de confiança para risco relativo.

\section{RESULTADOS}

Os dados relacionados ao sexo, idade e infecção pelo $H$. pylori, de ambos os grupos, encontram-se discriminados nas Tabelas 1,2 e 3 . Quanto à infecção pelo $H$. pylori, de acordo com a localização do tumor, foi demonstrado que os pacientes com lesões distais apresentam maior prevalência da infecção $(43,9 \%)$, quando comparados aos portadores de tumores da cárdia, cuja taxa foi de
12,5\% (Tabela-4). A Tabela-5 apresenta a distribuição dos pacientes infectados, correlacionando-os com a classificação de Borrmann. Verificou-se que 37,6\% dos adenocarcinomas gástricos apresentavam-se no nível III desta classificação e estavam infectados pelo H. pylori, contra $25 \%$ dos casos classificados no mesmo nível e que não estavam infectados pelo microorganismo (diferença não significativa). A análise de uma das variáveis pela medida de associação V de Cramer, demonstrou que existe uma associação significativa entre o grau de diferenciação histológica e a positividade do teste da urease $(\mathrm{p}<$ $0,10)$, como mostram os resultados nas Tabelas 6 e 7.

Não houve diferença significativa nos percentuais de positividade do teste da urease entre os pacientes portadores de câncer gástrico $(28,1 \%)$ e os pacientes do grupo controle $(25 \%)$, conforme demonstrado na Tabela-8.

Utilizando-se o modelo de regressão logística na avaliação de três variáveis (Tabelas 9 e 10),

Tabela 1 - Distribuição dos pacientes portadores de adenocarcinoma gástrico e controle por sexo e faixa etária.

\begin{tabular}{|c|c|c|c|c|c|c|c|}
\hline \multicolumn{2}{|c|}{ Grupos de Estudo } & \multicolumn{4}{|c|}{ Sexo } & \multicolumn{2}{|c|}{ Total } \\
\hline & & \multicolumn{2}{|c|}{ Masculino } & \multicolumn{2}{|c|}{ Feminino } & \multirow[b]{2}{*}{$\mathbf{n}$} & \multirow[b]{2}{*}{$\%$} \\
\hline & & $\mathbf{n}$ & $\%$ & $\mathbf{n}$ & $\%$ & & \\
\hline \multirow[t]{3}{*}{$\overline{C A}$ gástrico } & 20 a 39 & 2 & 12,5 & & & 2 & 12,5 \\
\hline & 40 a 59 & 4 & 25,0 & 1 & 6,2 & 5 & 31,2 \\
\hline & $\geq 60$ & 6 & 37,5 & 3 & 18,8 & 9 & 56,3 \\
\hline Total & & 12 & 75,0 & 4 & 25,0 & 16 & 100,0 \\
\hline \multirow[t]{3}{*}{ Controles } & 20 a 39 & & & 1 & 6,2 & 1 & 6,2 \\
\hline & 40 a 59 & 8 & 50,0 & 4 & 25,0 & 12 & 75,0 \\
\hline & $\geq 60$ & 3 & 18,8 & & & 3 & 18,8 \\
\hline Total & & 11 & 68,8 & 5 & 31,2 & 16 & 100,0 \\
\hline
\end{tabular}

Tabela 2 - Distribuição dos pacientes portadores de câncer gástrico e controles infectados ou não pelo Helicobacter pylori por faixa etária.

\begin{tabular}{|c|c|c|c|c|c|c|}
\hline \multirow[t]{3}{*}{ Faixa etária (anos) } & \multicolumn{4}{|c|}{ Teste da Urease } & & \\
\hline & \multicolumn{2}{|c|}{ Positivo } & \multicolumn{2}{|c|}{ Negativo } & \multicolumn{2}{|c|}{ Total } \\
\hline & $\mathbf{n}$ & $\%$ & $\mathbf{n}$ & $\%$ & $\mathbf{n}$ & $\%$ \\
\hline 20 a 39 & 1 & 3,1 & 2 & 6,3 & 3 & 9,4 \\
\hline 40 a 59 & 8 & 25,0 & 9 & 28,1 & 17 & 53,1 \\
\hline$\geq 60$ & 8 & 25,0 & 4 & 12,5 & 12 & 37,5 \\
\hline Total & 17 & 53,1 & 15 & 46,9 & 32 & 100,0 \\
\hline
\end{tabular}


Tabela 3 - Distribuição dos pacientes portadores de câncer gástrico e controles infectados ou não pelo Helicobacter pylori por sexo.

\begin{tabular}{lrrrrrrr}
\hline Sexo & \multicolumn{2}{c}{ Teste da Urease } & \multicolumn{2}{c}{ Total } \\
& \multicolumn{2}{c}{ Positivo } & \multicolumn{2}{c}{ Negativo } & \multicolumn{2}{c}{ n } & \% \\
\hline Masculino & $\mathbf{n}$ & $\boldsymbol{\%}$ & $\mathbf{n}$ & $\boldsymbol{\%}$ & \multicolumn{1}{c}{ n } & 72,0 \\
Feminino & 10 & 31,2 & 13 & 40,8 & 23 & 28,0 \\
Total & 7 & 21,8 & 2 & 6,2 & 9 & 100,0 \\
\hline
\end{tabular}

Tabela 4 - Distribuição dos pacientes portadores de câncer infectados ou não pelo Helicobacter pylori por localização do tumor.

\begin{tabular}{lccccccc}
\hline $\begin{array}{l}\text { Localização do } \\
\text { tumor }\end{array}$ & \multicolumn{2}{c}{ Positivo } & \multicolumn{2}{c}{ Teste da Urease } & \multicolumn{2}{c}{ Nogativo } & \multicolumn{2}{c}{ Total } \\
& $\mathbf{n}$ & $\mathbf{\%}$ & $\mathbf{n}$ & $\mathbf{\%}$ & $\mathbf{n}$ & $\boldsymbol{\%}$ \\
\hline Cárdia & 2 & 12,5 & 2 & 12,5 & 4 & 25,0 \\
Estômago distal* & 7 & 43,8 & 5 & 31,2 & 12 & 75,0 \\
Total & 9 & 56,3 & 7 & 43,7 & 16 & 100,0 \\
\hline
\end{tabular}

* todas as localizações no estômago, exceto o cárdia (fundo, corpo, antro e associações destas regiões).

Tabela 5 - Distribuição dos pacientes portadores de câncer infectados ou não pelo Helicobacter pylori pela classificação de Borrmann.

\begin{tabular}{|c|c|c|c|c|c|c|}
\hline \multirow{3}{*}{$\begin{array}{l}\text { Classificação } \\
\text { de Borrmann }\end{array}$} & \multicolumn{5}{|c|}{ Teste da Urease } & \\
\hline & \multicolumn{2}{|c|}{ Positivo } & \multicolumn{2}{|c|}{ Negativo } & \multicolumn{2}{|c|}{ Total } \\
\hline & $\mathbf{n}$ & $\%$ & $\mathbf{n}$ & $\%$ & $\mathbf{n}$ & $\%$ \\
\hline I & 0 & 0 & 2 & 12,5 & 2 & 12,5 \\
\hline II & 2 & 12,5 & 1 & 6,2 & 3 & 18,7 \\
\hline III & 6 & 37,6 & 4 & 25,0 & 10 & 62,6 \\
\hline IV & 1 & 6,2 & 0 & 0 & 1 & 6,2 \\
\hline Total & 9 & 56,3 & 7 & 43,7 & 16 & 100,0 \\
\hline
\end{tabular}

Tabela 6 - Distribuição dos pacientes portadores de câncer gástrico infectados ou não pelo Helicobacter pylori pelo grau histológico de diferenciação.

\begin{tabular}{|c|c|c|c|c|c|c|}
\hline \multirow{3}{*}{$\begin{array}{l}\text { Grau histológico } \\
\text { de diferenciação }\end{array}$} & \multicolumn{4}{|c|}{ Teste da Urease } & \multirow{2}{*}{\multicolumn{2}{|c|}{ Total }} \\
\hline & \multicolumn{2}{|c|}{ Positivo } & \multicolumn{2}{|c|}{ Negativo } & & \\
\hline & $\mathbf{n}$ & $\%$ & $\mathbf{n}$ & $\%$ & $\mathbf{n}$ & $\%$ \\
\hline Pouco dif. & 1 & 6,2 & 4 & 25,0 & 5 & 31,2 \\
\hline Moderadamente dif. & 6 & 37,6 & 3 & 18,7 & 9 & 56,3 \\
\hline Bem dif. & 2 & 12,5 & & & 2 & 12,5 \\
\hline Total & 9 & 56,3 & 7 & 43,7 & 16 & 100,0 \\
\hline
\end{tabular}


Tabela 7 - Medida da associação $V$ de Cramer e sua significância: associação entre grau de diferenciação do câncer gástrico e presença de infecção pelo Helicobacter pylori.

\begin{tabular}{ccc}
\hline Variáveis & V Cramer & Significância $(\mathbf{p})$ \\
\hline Grau de diferenciação & 0,537 & $0,099^{*}$ \\
$\mathrm{X}$ & & \\
Infecção pelo Helicobacter \\
pylori
\end{tabular}

* associação considerada significante quando $p<0,10$.

Tabela 8 - Distribuição da infecção por Helicobacter pylori em pacientes portadores de câncer gástrico e controles.

\begin{tabular}{lcccccc}
\hline \multicolumn{1}{c}{ Grupo de Estudo } & \multicolumn{2}{c}{ Teste da Urease } & \multicolumn{2}{c}{ Total } \\
& \multicolumn{2}{c}{ Positivo } & \multicolumn{2}{c}{ Negativo } & \multicolumn{2}{c}{} \\
& n & \% & n & \% & n & \% \\
\hline CA gástrico & 9 & 28,1 & 7 & 21,9 & 16 & 50,0 \\
Controle & 8 & 25,0 & 8 & 25,0 & 16 & 50,0 \\
Total & 17 & 53,1 & 15 & 46,9 & 32 & 100,0 \\
\hline
\end{tabular}

Tabela 9 - Estimativa do risco relativo para a ocorrência de câncer gástrico avaliando três variáveis (presença de infecção pelo Helicobacter pylori, sexo e idade) através do modelo de regressão logística.

\begin{tabular}{lccc}
\hline \multicolumn{1}{c}{ Variáveis } & Risco & Intervalo & Com 95\% de confiança \\
\hline Urease $(+)$ & 1,21 & 0,26 & 5,59 \\
Sexo & 0,64 & 0,12 & 3,46 \\
Idade & 0,96 & 0,90 & 1,02 \\
\hline
\end{tabular}

Tabela 10 - Classificação predita para os grupos experimental e controle com o modelo de regressão logística.

\begin{tabular}{lcc}
\hline Observado & \multicolumn{2}{c}{ Predito pela Regressão Logistica } \\
& Experimental & Controle \\
\hline CA gástrico & 11 & 5 \\
Controle & 5 & 11 \\
\hline
\end{tabular}

demonstrou-se que o fator de risco relativo de maior relevância para a ocorrência de câncer gástrico é a positividade do teste da urease (odds ratio $=1,28)$.

\section{DISCUSSÃO}

Embora a incidência e a mortalidade do câncer gástrico tenham declinado nas últimas décadas ${ }^{20,21}$, muitas pesquisas têm sido realizadas nos dias atuais, para investigar a participação do $H$. pylori nos processos inflamatórios e neoplásicos gástricos ${ }^{22,23}$. A grande maioria dos estudos é de sorologia para o $H$. pylori ${ }^{8}$ e muitos destes identificaram alta prevalência de anticorpos IgG antiH. pylori no soro dos pacientes, o que pode refletir estado de infecção prévia por este agente, em comparação com os grupos controle ${ }^{23-25}$. 
Diversos métodos para o diagnóstico da infecção pelo $H$. pylori são atualmente descritos ${ }^{4,15,16}$ : cultura, microscopia, determinação da atividade ureásica pelo teste respiratório ou através de fragmento de mucosa gástrica (teste da urease), detecção do DNA do microorganismo por PCR (polymerase chain reaction) e detecção do anticorpo IgG por ELISA e Western Blot. Todos são eficientes no diagnóstico da infecção, sendo alta a especificidade, enquanto que a sensibilidade é mais variável ${ }^{17}$. No entanto, os altos custos relativos à realização dos métodos mais avançados como os teste sorológicos, aliados à falta de viabilidade na prática médica diária fez com que optássemos pelo teste da urease, realizado através dos fragmentos gástricos obtidos no momento do exame endoscópico. Este método é seguro e prático, e possibilita o diagnóstico definitivo da infeção ${ }^{17}$, além de contar com a vantagem da visualização endoscópica simultânea das lesões gástricas.

Um total de 16 pacientes foram avaliados segundo idade, sexo, localização do tumor, classificação de Borrmann e grau de diferenciação celular. Todos os cânceres gástricos eram adenocarcinomas. A idade média do grupo experimental foi de 58,3 anos (variação de 35 a 75 anos) e do grupo controle de 52,5 anos (variação de 38 a 81 anos).

Na Tabela 1, observa-se que a maior parte dos pacientes portadores de adenocarcinoma gástrico situava-se na faixa etária acima dos 59 anos de idade e era do sexo masculino (3:1 em relação ao feminino). Estes dados estão de acordo com outras publicações da literatura especializada quanto à epidemiologia do câncer gástrico ${ }^{8}$.

O percentual de positividade para o teste da urease nos portadores de câncer gástrico foi de 28,1\% versus $25 \%$ do grupo controle (Tabela 8). Apesar de tratar-se de um estudo com casuística pequena, em estudo na Coréia, Kim et al ${ }^{26}$ observaram igualmente uma pequena diferença entre os pacientes portadores de câncer gástrico $(60 \%)$ e os controles $(51,9 \%)$.

Embora neste estudo não tenha sido observada associação estatisticamente significante entre infecção pelo $H$. pylori e adenocarcinoma gástrico, existe risco relativo estimado em 1,28 nesta associação (odds ratio=1,28). Ou seja, a ocorrência do câncer gástrico é 1,28 vezes maior nos pacientes infectados pelo $H$. pylori. Trata-se de um valor relativo baixo, comparado com outras publicações do gênero onde o risco atingiu o índice de 7,03 ( $p<0,0005)^{7}$. Estudos de sorologia demonstraram estimativas de risco para a ocorrência do câncer gástrico de 1,04 $(0,73-1,49)^{27}$, até de $0,54(0,24-1,2)^{28}$, o que revela que os dados da literatura não são uniformes. Entretanto, vale ressaltar que a maior parte desses estudos indica que o $H$. pylori é um importante fator de risco para o câncer gástrico (odds ratio=1,92, 1,322,78, com intervalo de confiança de $95 \%)^{8}$.

Quanto à idade (Tabela 2), observamos que cerca de $50 \%$ dos pacientes infectados pelo $H$. pylori tinham 40 anos ou mais, não se observando nenhuma diferença percentual entre as faixas etárias de 40 a 59 anos e ${ }^{3} 60$ anos. O sexo masculino apresentou prevalência da infecção de $31,3 \%$ comparada a $21,9 \%$ do sexo feminino (Tabela 3), dados semelhantes aos encontrados na maioria dos trabalhos da literatura ${ }^{8}$.

De acordo com nossos resultados, os pacientes com tumores de localização distal (não relacionados à cárdia) apresentavam maior prevalência de infecção (43,8\% versus $12,5 \%)$. O antro gástrico foi o local mais freqüente de apresentação dos tumores. A associação entre a infecção pelo $H$. pylori e os tumores gástricos distais foi bem documentada na literatura, embora os resultados não sejam uniformes e variarem entre os diversos estudos ${ }^{29}$.

Segundo a classificação de Borrmann, os tumores III e IV têm pior prognóstico por serem considerados tumores de grau mais avançado, associando-se a uma probabilidade maior de doença metastática e menor possibilidade de cura por ressecção. No presente trabalho, 37,6\% dos cânceres de estômago (Tabela 5) apresentavam-se no nível III (úlcero-infiltrativo) desta classificação e encontravam-se infectados pelo $H$. pylori, contra $25,0 \%$ dos pacientes que apresentavam o mesmo nível na classificação de Borrmann e não estavam infectados pelo microorganismo.

Histologicamente, os adenocarcinomas gástricos podem ser classificados em pouco, moderadamente ou bem diferenciados. Os tumores pouco diferenciados são mais agressivos por apresentarem graus de atipias mais intensos. Nos pacientes portadores de adenocarcinoma de moderado grau de diferenciação, 37,6\% estavam infectados pelo H. pylori versus $18,7 \%$ daqueles que possuíam o mesmo grau de diferenciação e não estavam infectados (Tabela 6). Pela medida de associação V de Cramer, existe uma associação significativa entre 
o grau de diferenciação histológica e a positividade do teste da urease $(p<0,10)$. Numa escala de 0 a 1 , esta associação é quantificada em 0,537 (Tabela-7).

Utilizou-se o modelo de regressão logística para avaliar os fatores de risco de infecção pelo $H$. pylori, sexo e idade na ocorrência do câncer gástrico. A Tabela-9 apresenta o risco em um intervalo com $95 \%$ de confiança. Assim, o fator relativo de maior relevância para a ocorrência do câncer gástrico é a positividade do teste da urease. A parte preditiva do modelo utilizado possui um acerto de $68,75 \%$ dos casos analisados, conforme demonstra a Tabela-10. O modelo reconheceu 11 casos de câncer gástrico e 11 casos de indivíduos normais, acertando a classificação de 22 pacientes em um total de 32 , ou seja, em $68,75 \%$ dos diagnósticos de câncer gástrico baseados na presença da infecção pelo $H$. pylori, sexo e idade estariam corretos. Segundo este modelo, a presença da infecção por este microorganismo é o principal fator preditivo na ocorrência do adenocarcinoma gástrico.

Não foram encontradas diferenças estatisticamente significantes entre infecção pelo $H$. pylori e o sexo, idade, localização tumoral e o nível na classificação de Borrmann, entre os pacientes analisados. Estes achados podem estar relacionados ao tamanho da amostra e a fatores de confundimento.
Pelo exposto, podemos concluir que a infecção pelo $H$. pylori foi mais prevalente em indivíduos com a idade $\geq 40$ anos e do sexo masculino. A infecção foi mais freqüente em tumores de localização distal; o nível III (úlcero-infiltrante) da classificação de Borrmann apresentou maiores percentuais de infecção pela bactéria; o percentual de infecção em pacientes com câncer gástrico foi mais elevado, em números absolutos, que nos pacientes do grupo controle. $\mathrm{O}$ risco relativo da ocorrência da neoplasia gástrica em pacientes infectados pelo $H$. pylori é baixo ${ }^{1,28}$, o que pode estar relacionado ao tamanho da amostra ou a fatores de confundimento não determinados. A infecção pelo H. pylori é o principal fator preditivo na ocorrência do adenocarcinoma gástrico. Não houve diferença estatística significante entre a presença da infeção pelo $H$. pylori e o sexo, idade, localização do tumoral e o nível na classificação de Borrmann.

Assim, a determinação da prevalência da infecção pelo $H$. pylori e sua associação com o câncer gástrico necessitam ser melhor avaliadas, principalmente em regiões mais carentes. Estudos de seguimento com maior casuística são necessários para que seja possível dimensionar efetivamente o papel do H. pylori nos processos neoplásicos do estômago.

\begin{abstract}
Background: In recent years evidence of the association between Helicobacter pylori and gastric cancer has been related by many studies. This work aims both at investigating the prevalence of infection by this micro-organism in patients with gastric cancer from Cancer Hospital Napoleão Laureano (João Pessoa-PB) and at determining the relative risk to the development of this neoplasm in infected patients. Methods: Sixteen patients with confirmed diagnostic of gastric adenocarcinoma were submited to a high digestive endoscopy so that fragments of the stomach were paired with a group of sixteen controls with normal endoscopic exams. Results: Of the sixteen patients with cancer, 28,1\% were infected versus $25 \%$ of the control group. Within infected patients, H. pylori was more prevalent among those with distal gastric lesions (43,8\%), in Borrmann III (37,6\%) and moderately differentiated tumors(37,6\%). There was a statistically significant association between the degree of differentiation and the presence of the infection by the $\mathrm{H}$. Pylori $(p<0,10)$. The estimated relative risk of the association between gastric cancer and $\mathrm{H}$. Pylori infection was 1,28\% (odds ratio $=1,28 \%$ ). Conclusion: Based on these results we can conclude that $\mathrm{H}$. Pylori infection is a factor of relative risk for the development of gastric adenocarcinoma.
\end{abstract}

Key words: Helicobacter pylori; Gastric cancer; Infection; Prevalence. 


\section{REFERÊNCIAS}

1. Palli D - Gastric cancer and Helicobacter pylori: a critical evaluation of the epidemiological evidence. Helicobacter, 1997, 2 (Suppl 1): S50-5.

2. Muñoz N, Franceschi S - Epidemiology of gastric cancer and perspectives for prevention. Salud Publica Mex, 1997, 39(4): 318-38.

3. Castro LP, Oliveira CA, Andrade JM - "Gastrites". In Castro LP, Dani R (eds) - Gastroenterologia Clínica. $3^{a}$ edição. Rio de Janeiro. Guanabara Koogan, 1993, pp. 562-585.

4. Butov Iul, Sadchikov VD, Khusnutdinov ShM, Kharchenko AV - Helicobacter pylori and stomach cancer. Lik Sprava, 1998, 220 (4): 69-71.

5. Wang JT, Chang CS, Lee CZ, Yang JC, Lin JT, Wang $\mathrm{TH}$ - Antibody to Helicobacter pylori species specific antigen in patients with adenocarcinoma of the stomach. Biochem Biophys Res Commun, 1998, 244 (2): 360-363.

6. Kim HY, Kim YB, Park CK, Yoo JY, Graham DY Co-existing gastric cancer and duodenal ulcer disease: role of Helicobacter pylori infection. Helicobacter, 1997, 2(4): 205-9.

7. Tanida N, Sakagami T, Sawada Y, Shimoyama T - Critical review of the WHO/IARC report regarding carcinogenicity of Helicobacter pylori. Nippn Rinsho, 1997, 55(4): 995-1002.

8. Huang J, Sridhar S, Chen Y, Hunt RH - Meta-analisis of the relationship between Helicobacter pylori seropositivity and gastric cancer. Gastroenterology, 1998, 114(4): 1169-1179.

9. Debongnie JC, Burette A, Glupezynski Y, Prez CDe, Koninck XDe, Donnay M - Helicobacter pylori and gastric cancer. An endoscopic series. Acta Gastro Enterologica Belgica, 1997, 60(3): 189-191.

10. Shibata T, Imoto I, Gabazza EC - Detection of Helicobacter pylori in biopsy of patients with gastric carcinoma. Biomed \& Pharmacother, 1997, 51(1): 2228.

11. Asaka M, Kudo M, Kato M, Sugiyama T, Takeda H Review article: Long-term Helicobacter pylori infection - from gastritis to cancer. Aliment Pharmacol Ther, 1998, 12 (Suppl 1): 9-15.

12. Torres J, Pérez Pérez GI - Infection with CagA Helicobacter pylori strains as a possible predictor of risk in the development of gastric adenocarcinoma in Mexico. Int J Cancer, 1998, 78(3): 298300.

13. Evans DG, Quiroz DM, Queiroz DM, Mendes EN, Evans DJJr - Helicobacter pylori CagA status and s and $\mathrm{m}$ alleles of vacA in isolates from individuals with a variety of $\mathrm{H}$. pylori-associated gastric diseases. J Clin Microbiol, 1998, 36(11): 3435-7.
14. Parsonnet J, Friedman GD, Orentreich N, Volgelman $\mathrm{H}$ - Risk for gastric cancer in people with CagA positive and Cag A negative Helicobacter pylori infection. Gut, 1997, 40(3): 297-301.

15. Elitsur Y, Hill I, Lichtman SN, Rosenberg AJ - Prospective comparison of rapid urease tests (PyloriTek, CLO test) for the diagnosis of Helicobacter pylori infection in symptomatic children: a pediatric multicenter study. Am J Gastroenterol, 1998, 93(2): 217-9.

16. Sternberg A, Coscas D, Wagner Y, Auslander L, Kaufshtein M, Fireman Z - Comparison of various Helicobacter pylori detection methods: serology, histology and Bacteriology. Irs J Med Sci, 1998, 33(3): 160-3.

17. Cohen H, Laine L - Endoscopic methods for the diagnosis of Helicobacter pylori. Aliment Pharmacol Ther, 1997, 11(Suppl 1): 3-9.

18. Asaka M, Takeda H, Sugiyama T, Kato M - What role does Helicobacter pylori play in gastric cancer? Gastroenterology, 1997, 113(Suppl 6): S56-60.

19. Coelho LVG - "Helicobacter pylori e doenças gastroduodenais". In Mincis M (ed) - Gastroenterologia e hepatologia: diagnóstico e tratamento. $2^{\text {a }}$ edição. São Paulo. Lemos Editorial, 1998. pp. 313332.

20. Parkin DM, Pisani P, Ferlay J - Estimates of the worlwide incidence of eighteen major cancers in 1985. Int J Cancer, 1993, 54(3): 594-606.

21. Coggon D, Acheson ED - The geography of cancer of the stomach. Br Med Bull, 1984, 49(2): 335-341.

22. Correa P, Haenezel W, Cuello C, Zavala D, Fontham E, Zarama G, Tannenbaum S, Collazos T, Ruiz B Gastric precancerous process in a high risk population: cohort follow up. Cancer Res, 1990, 50(7): 4737-4740.

23. Crabtree JE, Wyatt JI, Sobala GM, Miller G, Tompkins DS, Prinrose JN, Morgan AG - Systemic and mucosal humoral responses to Helicobacter pylori in gastric cancer. Gut, 1993, 34(3): 1339-1343.

24. Forman D, Sitas F, Newell DG, Stacey AR, Boreham J, Peto R, Campbell TC, Lee J, Chen J - Geographic association of Helicobacter pylori antibody prevalence and gastric cancer mortality in rural China. Int J Cancer, 1990, 46(3): 608-611.

25. Correa P, Fox J, Fontham E, Ruiz B, Lin Y, Zavala D, Taylor N, Mackinley D, Lima ED, Portilla H, Zarama G - Helicobacter pylori and gastric carcinoma: serum antibody prevalence in populations with contracting cancer risks. Cancer, 1990, 66(4): 2569-2574.

26. Kin HY, Cho BD, Chang WK, Kin DJ, Kin YB, Park CK, Chin HS, Yoo JY - Helicobacter pylori infection and the risk of gastric cancer among the Korean population. J Gastroenterol Hepatol, 1997, 12(1): 100-103. 
27. Fukuda H, Saito D, Hayashi S, Hisai H, Ono H, Yoshida S, Oguro Y, Noda T, Sato T, Katoh M, Terada M, Sugimora T - Helicobacter pylori infection, serum pepsinogen level and gastric cancer: a case-control study in Japan. Jpn J Cancer Res, 1995, 86(1): 64-71.

28. Stevens J, Fidalgo P, Tendeiro T, Chagas C, Ferra A, Nobre Leitao C, Costa Mira F - Anti- Helicobacter pylori antibodies prevalence and gastric adenocarcinoma in Portugal: report of a case-control study. Eur J Cancer Prevent, 1993, 2(2): 377-380.
29. Kikushi S, Wada O, Nakajima T, Nishi T, Kobayashi O, Konishi T, Ibana Y and The Research Group on Prevention of Gastric Carcinoma Amorng Young Adults - Serum anti-Helicobacter pylori antibody and gastric carcinoma among young adults. Cancer, 1995, 75(6): 2789-2793.

Endereço para correspondência:

Dr. Marcelo Fernandes Rangel, TCBC

R. Clodoaldo Gouveia, 66 - Centro

58013-370 - João Pessoa - PB

E-mail: mfernandesrangel@bol.com.br 